lustitia Socialis. Revista Arbitrada de Ciencias Jurídicas.

Año V. Vol. V. №3. Edición Especial. 2020-III:

Universidad Regional Autónoma de los Andes

Hecho el depósito de Ley: FA2016000064

ISSN: 2542-3371

FUNDACIÓN KOINONIA (F.K). Santa Ana de Coro, Venezuela

Julián Rodolfo Santillán-Andrade; Jean Carlos Espinoza-Olguín

http://dx.doi.org/10.35381/racii.v5i3.1128

\title{
Estado de embriaguez como agravante en delitos que atentan contra la integridad de las personas
}

Drunkenness as aggravating in crimes that violate the integrity of people

\author{
Julián Rodolfo Santillán-Andrade \\ us.juliansantillan@uniandes.edu.ec \\ Universidad Regional Autónoma de los Andes, Santo Domingo \\ Ecuador \\ https://orcid.org/0000-0002-2614-5676 \\ Jean Carlos Espinoza-Olguín \\ us.jeanespinosa@uniandes.edu.ec \\ Universidad Regional Autónoma de los Andes, Santo Domingo \\ Ecuador
}

Recibido: 31 de octubre de 2020

Revisado: 10 de octubre de 2020

Aprobado: 05 de diciembre de 2020

Publicado: 10 de diciembre de 2020 


\title{
RESUMEN
}

La investigación se realizó desde la perspectiva cuantitativa, con una metodología descriptiva y un diseño no experimental, apoyándose en el análisis documental y bibliográfico lo que permitió explorar documentos, normas, leyes, investigaciones afines con el tema. El objetivo del presente trabajo es determinar el agravante como delito vehicular contra la integridad de las personas producto del estado de embriaguez. La población con la que se trabajó esta investigación fueron aplicado a operadores del derecho conocedores del tema, como Jueces, Fiscales y Abogados quienes expusieron sus opiniones sobre la cuestión de la embriaguez y la agravación de la pena en delitos. Estas fueron hechas a través de medios de comunicación, vía telefónica, mensajería instantánea y correos electrónicos. Se concluye que debe agravarse la pena al conductor cuando conduciendo en estado de embriaguez, ocasione la muerte a una o varias personas; y, por otro lado, constituyendo la ingestión de bebidas alcohólicas (alcoholismo) un problema cultural.

Descriptores: Bebida alcohólica; derecho penal; adicción. (Palabras tomadas del Tesauro UNESCO).

\begin{abstract}
The research was carried out from a quantitative perspective, with a descriptive methodology and a non-experimental design, relying on documentary and bibliographic analysis, which allowed the exploration of documents, norms, laws, and research related to the subject. The objective of this work is to determine the aggravating factor as a vehicular crime against the integrity of people as a result of the state of intoxication. The population with whom this investigation was worked was applied to legal operators familiar with the subject, such as Judges, Prosecutors and Lawyers who presented their opinions on the issue of drunkenness and the aggravation of the penalty in crimes. These were done through the media, by phone, instant messaging and emails. It is concluded that the penalty to the driver should be aggravated when driving while intoxicated, causes the death of one or more people; and, on the other hand, constituting the ingestion of alcoholic beverages (alcoholism) a cultural problem.
\end{abstract}

Descriptors: Alcoholic beverage; criminal law; addiction. (Words taken from the UNESCO Thesaurus). 
lustitia Socialis. Revista Arbitrada de Ciencias Jurídicas.

Año V. Vol. V. №3. Edición Especial. 2020-III:

Universidad Regional Autónoma de los Andes

Hecho el depósito de Ley: FA2016000064

ISSN: 2542-3371

FUNDACIÓN KOINONIA (F.K). Santa Ana de Coro, Venezuela

Julián Rodolfo Santillán-Andrade; Jean Carlos Espinoza-Olguín

\section{INTRODUCCIÓN}

A la embriaguez se le puede definir como el conjunto de alteraciones o trastornos psicofísicos que sufre una persona como consecuencia de la ingestión excesiva de bebidas alcohólicas. Este concepto ha sido reconocido por la práctica judicial, que al respecto afirma que el concepto jurídico de la embriaguez coincide, dada su simplicidad, con el valor léxico del vocablo, pues éste sólo significa un trastorno temporal de las potencias anímicas que produce el uso excesivo de las bebidas alcohólicas (Real Academia Española, 2019).

La imagen de la embriaguez es siempre una concepción relativa, deberá ser puesto en relación comparativa con las particulares condiciones fisio-psíquicas del procesado. Por eso el volumen de alcohol bebido no posee por sí solo todo el valor concluyente. Si en el fallo no se establece el nivel en que el alcohol ingerido, afectó al figurante del suceso, hay que concebir que el juzgado no estimó probado más que el hecho de la ingestión de bebidas alcohólicas por él.

El instante en el cual debe valorarse el nivel de embriaguez, en el sentido de la ley, es aquel en que la persona ha ejecutado el acto sancionable, lo que es obligatorio valorar por ser la embriaguez un suceso continuo, que el tribunal, en su impulso, debe establecer el estadio donde tiene relación directa con la ecuación del ilícito. Por ello, el grado de enajenación que se haya alcanzado después de la consumación de la infracción no reemplaza la embriaguez preliminar y análoga, sobre la que deberá establecerse. Tampoco al cesar o atenuar la embriaguez por efectos de la emoción percibida al ejecutar el acto criminal o en virtud de sucesos ocurridos después de la consumación del mismo, no despoja al ilícito del casual agravamiento producto de la embriaguez anterior. Las justificaciones de prevención general se deducen de las alusiones que se hacían a la necesidad de motivar a los ciudadanos para que se abstuvieran de conducir bajo el influjo de bebidas embriagantes o de sustancias estupefacientes, por ser comportamientos considerados altamente peligrosos, consideraciones que en general se encontraban referidas a supuestos de homicidio imprudente en accidentes de tránsito. (Sotomayor-Acosta \& Álvarez-Álvarez, 2014:131) 
Desde la óptica legal existen dos polos, el que representa el "alcoholismo como enfermedad", con claro componente patológico de alteración de la personalidad, y "la simple embriaguez circunstancial”, en una escala que atiende tanto a su etiología como a los efectos psicológicos engendrados en el sujeto, asimilando su estado al propio trastorno mental transitorio, quedando en un estado estimativo inferior por afectación de las facultades volitivas e intelectivas. Aunque se ha tomado conciencia al respecto a pesar de que hace ya más de un siglo que los alcohólicos son considerados como enfermos, aún muchas personas los califican como perversos y los hacen objeto de marcada hostilidad, catalogándolos de modo despectivo como viciosos, y esta percepción, errónea aún, tiene arraigo en el ámbito forense, limitando en muchos casos el análisis objetivo y racional de la conducta imputada a los sujetos que han cometido delitos bajo los efectos de la ingestión de bebidas alcohólicas o la de quienes producto a su frecuente consumo se comportan indebidamente.

Respecto a este último supuesto, conviene demostrar la necesidad de distinguir entre conducta propiamente antisocial y toxicomanía, a los efectos de calificar correctamente el grado de peligrosidad en el que pudiera estar enmarcado el sujeto. El estado de embriaguez se constituye en una de las principales agravantes en la comisión de hechos delictivos que atentan contra la integridad de las personas, con especial repercusión en los incidentes de tráfico. La conducción de un vehículo automotor es un riesgo permitido frente al cual los conductores deberán observar reglas obligatorias durante la conducción; la ingestión de bebida alcohólica disminuye la capacidad de reacción del conductor, nublan su juicio y hacen que progresivamente pierda la conciencia. Por otro lado, está el individuo que voluntariamente se coloca en estado de embriaguez para cometer un hecho delictivo. Los adultos jóvenes de entre 20 y 29 años están expuestos a un mayor riesgo (hasta tres veces superior) de sufrir las consecuencias de conducir bajo los efectos del alcohol en comparación con los conductores mayores de 30 años, cualquiera que sea el nivel de alcoholemia. De hecho, el alto riesgo para los conductores jóvenes y novatos ha hecho que muchos países apliquen los límites de alcoholemia con más rigor en este grupo de población. También es importante destacar que la ingesta de alcohol no es solo un factor de 
riesgo para las personas que conducen automóviles y otros vehículos grandes, sino también para los demás usuarios de las vías. Algunos estudios realizados en países de bajos y medianos ingresos revelaron la presencia de alcohol en la sangre en entre el $18 \%$ y el $90 \%$ de los peatones y entre el $10 \%$ y el $28 \%$ de los motociclistas que sufrieron lesiones en siniestros viales. (Organización Panamericana de la Salud, 2107:3)

Las infracciones de tránsito continúan siendo una de las principales causas de accidentabilidad, Según las estimaciones del informe sobre la situación mundial en seguridad vial de la Organización Mundial de la Salud, publicado en el 2015, Ecuador ocupa el séptimo puesto en Latinoamérica en tasa de fallecimientos por accidentes de tránsito y el décimo tercero a escala, global. (Algora-Buenafé \& Otros,2017:54), denotándose la falta de previsión y responsabilidad en muchos conductores, haciéndose cada vez más temeraria su actuación en ese estado, por tantos daños, lesiones y muertes que ocasionan; sin embargo, en el Código Orgánico Integral Penal no se verifica la agravación de la pena cuando por ello se causa la muerte a otro, el marco sancionador permanece inalterable. existe un alto índice de accidentes de tránsito provocado por los conductores en estado de embriaguez, la falta de recursos, control y de educación provoca que los conductores se encuentren involucrados en los accidentes de tránsito, estos no miden el peligro en los que se encuentran vinculados al conducir un vehículo en estado de embriaguez, tampoco establecen la problemática que lleva consigo, y a quienes afectaría su impericia, no toman en cuenta que no solo pueden ser ellos los afectados sino que también a las personas que los rodean y a sus familiares. (Cruz-Piza \& Otros, 2020:126)

De aquí que el tema tenga actualidad e importancia, pues con él será posible demostrar la necesidad de modificar el Código Orgánico Integral Penal, no tanto con la finalidad de castigar a quienes incurren en tan peligrosa conducta, sino más bien para prevenir la ocurrencia de eventos fatales.

El objetivo del presente trabajo es determinar el agravante como delito vehicular contra la integridad de las personas producto del estado de embriaguez. 


\section{METODOLOGÍA}

La investigación se realizó desde la perspectiva cuantitativa, con una metodología descriptiva y un diseño no experimental, apoyándose en el análisis documental y bibliográfico lo que permitió explorar documentos, normas, leyes, investigaciones afines con el tema. Permitiendo establecer mediante el método analítico-sintético, la evidencia teórico jurídico relacionado al tema. Para la compilación de los datos se realizó mediante una encuesta y se aplicó un cuestionario. La población con la que se trabajó esta investigación fueron aplicado a operadores del derecho conocedores del tema, como Jueces, Fiscales y Abogados, quienes expusieron sus opiniones sobre la cuestión de la embriaguez y la agravación de la pena en delitos que lesionan la integridad de los individuos. Estas fueron hechas a través de medios de comunicación, tales como vía telefónica, mensajería instantánea y correos electrónicos.

\section{RESULTADOS}

Se examinaron las principales normas jurídicas que abordan la cuestión del estado de embriaguez; se partió del análisis del reglamento a la norma orgánica de transporte terrestre, donde se verificó especialmente la definición y alcance del estado embriaguez.

Por otro lado, se tuvo a la vista el Código Orgánico Integral Penal, respecto del cual se verificó lo relativo a las infracciones de tránsito, y su tratamiento, especialmente en lo relativo a las agravantes de la responsabilidad penal.

Se analizaron y compararon otras normativas del ordenamiento jurídico con otras normas y doctrinas de otras latitudes del continente, sin que la norma vigente esté en consonancia con la tendencia de muchos países de considerar al estado de embriaguez como agravante de la responsabilidad penal en cualquiera de sus manifestaciones, siempre que se conciba como determinante de la conducta delictiva. Con la finalidad de fundamentar presupuestos teóricos sobre el estado de embriaguez como agravante en los delitos que atentan contra la integridad de las personas, se encuestaron a los jueces Javier Martínez, Wilson Bolívar, José Luís Alvarado, Igor Vasco, Patricio Calderón, Max Salazar, Mario Fuentes, Alex Samaniego, Glendy 
Zambrano y Julia Leiva, los que al presentársele el cuestionario elaborado a esos efectos respondieron como sigue:

La primera interrogante estuvo dirigida a conocer acerca de las obligaciones de un conductor involucrado en accidente de tránsito cuando ocasiona lesiones o la muerte de alguien; por el mismo orden en que se relacionan los entrevistados, se obtuvieron diversas respuestas; el primero expuso que el conductor está obligado a detenerse, prestar ayuda y dar cuenta a la autoridad que corresponda a los efectos de la denuncia judicial; otro entrevistado se refirió únicamente a la reparación integral de la víctima; otro alega la prestación de auxilio, traslado a una casa de salud y comunicarlo al 911 u órgano auxiliar más cercano, mientras que el cuarto de los entrevistados refiere la reparación integral por daños. Es que en la mayoría de los accidentes el infractor se retira, haciéndose imposible la práctica de pruebas, especialmente la toxicológica para determinar el porciento de alcohol en sangre.

En segundo lugar, se preguntó ¿qué pasaría si el conductor se retirara del lugar o no da cuenta a la autoridad policial en caso de accidente? Aquí el primero los entrevistados se refiere a los supuestos en los que se causan daños, lesiones o la muerte de una persona, señalando la pena que procede en cada uno; el segundo plantea que constituye una agravante, pero en caso de que no sea capturado, se salva de ir preso; el tercero y el cuarto de los entrevistados lo admiten como agravante a tomar en cuenta en el proceso. De igual manera, si el conductor se sustrae del proceso lograría su impunidad, o al menos ocultar el estado de embriaguez si es que ha ingerido bebidas alcohólicas.

La tercera interrogante, ¿puede un conductor negarse a que le practiquen la prueba respiratoria o de otra naturaleza para comprobar la presencia de alcohol u otra sustancia en su sangre; tiene esto que ver con su autoincriminación; y qué pasa en caso de negarse? El primer entrevistado solo refirió que en tal circunstancia será sancionado con multa de diez unidades tributarias mensuales y con la suspensión de la licencia de conducción hasta por un mes; el segundo plantea que sí puede negarse y que no tiene nada que ver; el tercero responde que no, pero se supondrá mediante la sana crítica y la costumbre y se aplicará el máximo de la embriaguez por litro de 
sangre; y el cuarto expone que si puede negarse, no siendo claro en su planteamiento; y en caso de negarse o realizar otra maniobra que altere el resultado de los exámenes recibirá la sanción correspondiente. Como se observa son diversos los criterios sobre la cuestión planteada, no se habla de agravantes, evidenciándose falencias que pueden trascender al fondo del asunto y por tanto a fallos arbitrarios.

La cuarta pregunta, ¿qué pena se aplica a las personas que en estado de ebriedad o bajo influencia de sustancias estupefacientes o sicotrópicas causas la muerte o lesiones graves a una persona? El primero, en el caso de lesiones se refiere a presidio de tres años y un día a cinco años, y, en caso de muerte, de tres años y un día a diez años, además de la suspensión perpetua para conducir y el comiso del vehículo. Los demás solo se limitaron a decir que se le aplica pena de die años d presidio. Como se observa ninguno de los entrevistados se refirió a la agravación.

En la quinta se preguntó sobre ¿qué agravantes tienen lugar cuando se produce un accidente en el que motivado al estado de embriaguez del conductor se producen lesiones o la muerte de alguna persona? En tal caso solo se limitan a expresar que será sancionado con el máximo de la pena correspondiente a la infracción cometida. Ninguno de los entrevistados hace referencia a alguna agravante, es que el hecho de que se imponga el máximo de la pena, no significa la agravación propiamente dicha, esto sería mal interpretar el verdadero sentido de lo que es una agravante, que no es otra cosa que aumentar los límites mínimos y máximos ya estipulados por el legislador, en le medida que corresponda.

La sexta interrogante se refiere a ¿cuál es el tiempo mínimo de cárcel efectiva para el conductor que en estado de embriaguez o bajo efecto de sustancias estupefacientes o sicotrópicas cause lesiones graves o la muerte de una persona para poder obtener su libertad? De manera unánime plantean que deberá cumplir dos tercios de la condena.

Y la séptima pregunta estuvo referida a ¿cómo valoran los jueces el hecho de que una persona, voluntariamente, se coloque en estado de embriaguez para cometer delito? En cuyo caso coinciden en que se aplica la pena prevista para el delito, sin que se 
considere a tal estado como atenuante o agravante, por cuanto era su intención desde antes de colocarse en dicho estado.

Con la misma finalidad se encuestaron diez abogados del libre ejercicio de la ciudad de Santo Domingo, por lo cual se elaboró la siguiente encuesta cuyo resultado fue el siguiente:

Tabla 1.

\begin{tabular}{|l|c|c|c|c|}
\hline \multicolumn{4}{|c|}{ ¿Conoce las contravenciones de tránsito establecidas en el Código } \\
Orgánico Integral Penal? \\
\hline \multirow{2}{*}{ Enunciados. } & \multicolumn{1}{|c|}{ Si } & \multicolumn{2}{c|}{ No } \\
\cline { 2 - 5 } & Cantidad & $\%$ & Cantidad & $\%$ \\
\hline Subtotal. & 10 & 100 & 0 & 0 \\
\hline Total. & \multicolumn{4}{|c}{10} \\
\hline$\%$ & 100 & \multicolumn{4}{|c}{} \\
\hline
\end{tabular}

Elaborado por: Espinoza-Olguín (2021).

Fuente: Encuesta a Jueces, Fiscales y Abogados.

Se puede observar que la primera interrogante fue la referida a si conocen las contravenciones de tránsito establecidas en el Código Orgánico Integral Penal, a lo que todos respondieron afirmativamente.

Tabla 2.

\begin{tabular}{|l|l|l|l|l|l|l|}
\hline $\begin{array}{l}\text { ¿Cómo calificaría la clasificación de las contravenciones de tránsito de } \\
\text { acuerdo a la gravedad de las mismas? }\end{array}$ \\
\hline Enunciados. & \multicolumn{2}{|l|}{ Satisfactoria } & Regular & \multicolumn{2}{l|}{$\begin{array}{l}\text { Nada } \\
\text { satisfactoria }\end{array}$} \\
\cline { 2 - 8 } & Cantidad & $\%$ & Cantidad & $\%$ & Cantidad & $\%$ \\
\hline Subtotal. & 2 & 20 & 8 & 80 & 0 & 0 \\
\hline Total. & 10 & & & & & \\
\hline$\%$ & 100 &
\end{tabular}

Elaborado por: Espinoza-Olguín (2021).

Fuente: Encuesta a Jueces, Fiscales y Abogados. 
La segunda consistió en calificar en satisfactoria, regular o nada satisfactoria las contravenciones de tránsito de acuerdo a la gravedad de las mismas, en cuyo sentido dos respondieron que satisfactoria y ocho regular.

Tabla 3.

\begin{tabular}{|l|c|c|c|c|}
\hline \multicolumn{3}{|c}{$\begin{array}{c}\text { ¿Conoces las agravantes aplicables a los casos de lesiones o muerte en } \\
\text { accidente de tránsito provocado por el estado de embriaguez del conductor? }\end{array}$} \\
\hline Enunciados. & \multicolumn{2}{|c|}{ Si } & \multicolumn{2}{c|}{ No } \\
\cline { 2 - 5 } & Cantidad & $\%$ & Cantidad & $\%$ \\
\hline Subtotal. & 5 & 50 & 5 & 50 \\
\hline Total. & \multicolumn{4}{|c|}{10} \\
\hline$\%$ & \multicolumn{4}{|c}{100} \\
\hline
\end{tabular}

Elaborado por: Espinoza-Olguín (2021).

Fuente: Encuesta a Jueces, Fiscales y Abogados.

En tercer lugar, se preguntó si conocen las agravantes aplicables a los casos de lesiones o muerte en accidentes de tránsito provocado por el conductor en estado de embriaguez, en cuyo caso cinco respondieron que sí y cinco que no.

Tabla 4.

\begin{tabular}{|l|c|c|c|c|c|c|}
\hline \multicolumn{7}{|c|}{$\begin{array}{c}\text { ¿Cómo denominaría el proceso de juzgamiento de las contravenciones de } \\
\text { tránsito, en virtud del debido proceso? }\end{array}$} \\
\hline Enunciados. & Satisfactoria & Regular & Nada satisfactoria \\
\cline { 2 - 7 } & Cantidad & $\%$ & Cantidad & $\%$ & Cantidad & $\%$ \\
\hline Subtotal. & 0 & 0 & 8 & 80 & 2 & 20 \\
\hline Total. & \multicolumn{7}{|c|}{10} & & \\
\hline$\%$ & \multicolumn{7}{|c|}{100} \\
\hline
\end{tabular}

Elaborado por: Espinoza-Olguín (2021).

Fuente: Encuesta a Jueces, Fiscales y Abogados.

En cuarto puesto, se le pidió que evaluara de satisfactorio, regular o nada satisfactorio el proceso de juzgamiento de las contravenciones de tránsito en virtud del debido proceso, de los que ocho lo hicieron de regular y dos nada satisfactorio. 
Tabla 5.

\begin{tabular}{|l|c|c|c|c|}
\hline \multicolumn{3}{|c|}{ ¿Considera que las sanciones establecidas para las contravenciones de } \\
tránsito son proporcionales?
\end{tabular}

Elaborado por: Espinoza-Olguín (2021).

Fuente: Encuesta a Jueces, Fiscales y Abogados.

Si considera que las sanciones establecidas para las contravenciones de tránsito son proporcionales, fue la quinta pregunta, en cuyo caso 8 respondieron afirmativamente y solo dos lo hicieron de manera contraria.

Tabla 6.

\begin{tabular}{|c|c|c|c|c|c|c|}
\hline \multicolumn{7}{|c|}{$\begin{array}{c}\text { ¿En virtud de la finalidad de rehabilitar, cómo denominaría las penas } \\
\text { establecidas en el Código Orgánico Integral Penal? }\end{array}$} \\
\hline \multirow[t]{2}{*}{ Enunciados. } & \multicolumn{2}{|c|}{ Satisfactoria } & \multicolumn{2}{|c|}{ Regular } & \multicolumn{2}{|c|}{$\begin{array}{c}\text { Nada } \\
\text { satisfactoria }\end{array}$} \\
\hline & Cantidad & $\%$ & Cantidad & $\%$ & Cantidad & $\%$ \\
\hline Subtotal. & 8 & 80 & 2 & 20 & 0 & 0 \\
\hline Total. & \multicolumn{6}{|c|}{10} \\
\hline$\%$ & \multicolumn{6}{|c|}{100} \\
\hline
\end{tabular}

Elaborado por: Espinoza-Olguín (2021).

Fuente: Encuesta a Jueces, Fiscales y Abogados.

Como sexta cuestión, centrado en la finalidad de la rehabilitación, se le preguntó cómo denominaría las penas establecidas en el Código Orgánico Integral Penal, teniendo las opciones satisfactorio, regulas y nada satisfactorio, en cuyo sentido ocho la consideraron satisfactorio y dos regular. 
Tabla 7.

\begin{tabular}{|l|c|c|c|c|}
\hline \multirow{2}{*}{$\begin{array}{l}\text { ¿A su parecer, representan las contravenciones de tránsito por conducir en } \\
\text { estado de embriaguez, una infracción verdaderamente grave y de daño real? }\end{array}$} \\
\hline Enunciados. & \multicolumn{2}{|c|}{ Si } & \multicolumn{2}{c|}{ No } \\
\cline { 2 - 5 } & Cantidad & $\%$ & Cantidad & $\%$ \\
\hline Subtotal. & 10 & 100 & 0 & 0 \\
\hline Total. & \multicolumn{4}{|c|}{10} \\
\hline$\%$ & \multicolumn{4}{|c|}{100} \\
\hline
\end{tabular}

Elaborado por: Espinoza-Olguín (2021).

Fuente: Encuesta a Jueces, Fiscales y Abogados.

La séptima, para conocer si las contravenciones de tránsito representan una infracción verdaderamente grave y de daño real, a la que de manera unánime respondieron que sí.

Tabla 8.

8. ¿Considera posible la aplicación de medidas alternativas a la privación de libertad en las contravenciones de tránsito por conducir en estado de embriaguez?

\begin{tabular}{|c|c|c|c|c|}
\hline \multirow[t]{2}{*}{ Enunciados. } & \multicolumn{2}{|c|}{$\mathrm{Si}$} & \multicolumn{2}{|c|}{ No } \\
\hline & Cantidad & $\%$ & Cantidad & $\%$ \\
\hline Subtotal. & 10 & 100 & 0 & 0 \\
\hline Total. & \multicolumn{4}{|c|}{10} \\
\hline$\%$ & \multicolumn{4}{|c|}{100} \\
\hline
\end{tabular}

Elaborado por: Espinoza-Olguín (2021).

Fuente: Encuesta a Jueces, Fiscales y Abogados.

En octavo puesto se le formuló la pregunta sobre si consideraba posible la aplicación de medidas alternativas a la privación de libertad en las contravenciones de tránsito por conducir en estado de embriaguez, y todos los encuestados respondieron afirmativamente. 
Tabla 9.

\begin{tabular}{|c|c|c|c|c|}
\hline \multicolumn{5}{|c|}{$\begin{array}{l}\text { ¿Cree usted que las sanciones establecidas para las contravenciones de } \\
\text { tránsito por conducir en estado de embriaguez, son proporcionales? }\end{array}$} \\
\hline \multirow[t]{2}{*}{ Enunciados. } & \multicolumn{2}{|c|}{$\mathbf{S i}$} & \multicolumn{2}{|c|}{ No } \\
\hline & Cantidad & $\%$ & Cantidad & $\%$ \\
\hline Subtotal. & 7 & 70 & 3 & 30 \\
\hline Total. & \multicolumn{4}{|c|}{10} \\
\hline$\%$ & \multicolumn{4}{|c|}{100} \\
\hline
\end{tabular}

Elaborado por: Espinoza-Olguín (2021).

Fuente: Encuesta a Jueces, Fiscales y Abogados.

La novena cuestión se dirigió a conocer si las medidas por contravenciones de tránsito por estado de embriaguez son proporcionales, en la que 7 respondieron que sí y 3 que no.

Tabla 10.

\begin{tabular}{|l|c|c|c|c|c|c|}
\hline \multicolumn{7}{|c|}{ ¿Cómo denominaría la escala valorativa de las contravenciones de tránsito } \\
por conducir en estado de embriaguez?
\end{tabular}

Elaborado por: Espinoza-Olguín (2021).

Fuente: Encuesta a Jueces, Fiscales y Abogados.

La décima pregunta se realizó con la intención de evaluar la escala valorativa de las contravenciones de tránsito por conducir en estado de embriaguez; ocho lo hicieron como bueno y dos de malo. 
Tabla 11.

\begin{tabular}{|l|c|c|c|c|}
\hline \multirow{2}{*}{$\begin{array}{l}\text { ¿Considera necesario realizar una reforma al Código Orgánico Integral Penal, para } \\
\text { la aplicación de penas alternativas, en las contravenciones de tránsito por conducir } \\
\text { en estado de embriaguez? }\end{array}$} \\
\hline Enunciados. & \multicolumn{2}{|c|}{ Si } & \multicolumn{2}{c|}{ No } \\
\cline { 2 - 5 } & Cantidad & $\%$ & Cantidad & $\%$ \\
\hline Subtotal. & 10 & 100 & 0 & 0 \\
\hline Total. & \multicolumn{3}{|c|}{10} \\
\hline$\%$ & \multicolumn{3}{|c|}{100} \\
\hline
\end{tabular}

Elaborado por: Espinoza-Olguín (2021).

Fuente: Encuesta a Jueces, Fiscales y Abogados.

Y como pregunta número once y última se le pidió consideración sobre si es necesario realizar reforma al Código Integral Penal para la aplicación de penas alternativas en contravenciones de tránsito por conducir en estado de embriaguez; todos respondieron que sí.

Como se puede observar existen opiniones opuestas respecto al estado de embriaguez en los conductores, sin embargo, admiten la necesidad de reformar el Código Orgánico integral Penal.

\section{DISCUSIÓN}

Un conductor ebrio en la carretera puede ser catalogado como un potencial asesino en serie, pues el hecho de conducir en ese estado por la vía pública y en presencia de multitudes personas, pone en riesgo inminente la vida de muchos seres humanos. Con la aplicación de los métodos investigativos ya anunciados, se logró realizar un adecuado análisis de las principales normas jurídicas que abordan la cuestión del estado de embriaguez respecto a lo que se investiga. Se partió del análisis del reglamento a la norma orgánica de transporte terrestre, específicamente el artículo 243, donde se define al estado de embriaguez como la "pérdida temporal o evidente disminución de las aptitudes físicas e intelectuales normales, producto del consumo de alcohol o sustancias estupefacientes, lo que le impide garantizar, sin riesgo, la 
conducción de cualquier tipo de vehículo" (Asamblea Nacional constituyente del Ecuador, 2016).

Se verificaron los artículos 37, 376, 384 y 385 de la norma penal (COIP), en los que se establece, respectivamente, la responsabilidad por embriaguez o intoxicación, estableciendo las reglas específicas para el juzgamiento de quienes hayan cometido un ilícito en ese estado, incluso para quienes calculadamente se enajenan para ejecutar el delito, en cuyo caso siempre se considerará agravante, pero se excluyen las infracciones de tránsito, a las que se le da un tratamiento diferente; sin embargo, la modalidad delictiva analizada es tratada en los artículos 376, 384 y 385, el primero para el conductor que causa la muerte a otra persona en estado de ebriedad o con efectos de sustancias narcóticas, psicotrópicas o preparados que las contengan; el segundo, para el que conduzca un vehículo bajo los efectos de tales sustancias, y el tercero (Art. 385) es donde se establecen las escalas a los efectos de fijar la pena, sin que en ningún caso se hable de agravación a pesar de constituir los accidentes de tránsito, por la ingestión de bebidas alcohólicas, una de las fundamentales causas de muerte a escala global y nacional (Asamblea Nacional República del Ecuador, 2014).

\section{CONCLUSIONES}

Un conductor ebrio convierte al vehículo en un arma letal, sin embargo, en el Código Orgánico Integral Penal, a pesar de constituir el estado de embriaguez una agravante en el caso de lesiones causadas por accidente de tránsito, conforme con el artículo 379 , no lo es ante la muerte, a pesar de que existen fundamentos suficientes que lo justifican.

En los accidentes de tránsito que ocasiona el conductor ebrio, si es que se pretende resolver la problemática, habrá que dejar de hablar de culpa y sí del dolo eventual; no más excusas con el "no querer" de la culpa, pues, lógicamente, una persona que no actúe con el deber objetivo de cuidado que le corresponde como conductor, deja las cosas al azar, por el hecho de que un ebrio no puede asegurar que evitará el resultado, lo que permite calificar la conducción de temeraria. 
Pueden ser varias las posibles respuestas a la problemática; de momento debe agravarse la pena al conductor cuando conduciendo en estado de embriaguez, ocasione la muerte a una o varias personas; $y$, por otro lado, constituyendo la ingestión de bebidas alcohólicas (alcoholismo) un problema cultural, hay que seguir combatiéndola y educar a las nuevas y mayores generaciones.

\section{FINANCIAMIENTO}

No monetario.

\section{AGRADECIMIENTO}

A la Universidad Regional Autónoma de los Andes; por motivar el desarrollo de la Investigación.

\section{REFERENCIAS CONSULTADAS}

Algora-Buenafé AF, Russo-Puga M, Suasnavas-Bermúdez PR, Merino-Salazar P, \& Gómez-García AR. (2017) Tendencias de los accidentes de tránsito en Ecuador: 2000-2015. [Trends in Traffic Accidents in Ecuador:2000-2015]. Rev Gerenc Polít Salud. 16 (33): 52-58. Recuperado de: https://doi.org/10.11144/Javeriana.rgps16-33.tate

Asamblea Nacional Constituyente de la República del Ecuador, (2008). Constitución de la República del Ecuador. Montecristi. Registro Oficial 449 de 20-oct-2008. Recuperado de https://n9.cl/sia

Asamblea Nacional Constituyente de la República del Ecuador (2008). Reglamento a la Ley de Transporte Terrestre Transito y Seguridad Vial. [ Regulations to the Law on Land Transit Transport and Road Safety] Decreto Ejecutivo 1196 Registro Suplemento 731 de 25-jun-2016 última modificación 14-nov-2016.

Asamblea Nacional de la República del Ecuador. (2014). Código Orgánico Integral Penal. [Comprehensive Organic Criminal Code]. Recuperado de https://n9.cl/g6sc

Cruz-Piza, I., Pozo-Lucio, J., \& Gómez-Naranjo, V. (2020) El estado de embriaguez y su incidencia en los accidentes de tránsito [The state of intoxication and its incidence in traffic accidents]. IUSTITIA SOCIALIS.5(3), 124-133. Recupedarado de: $\underline{\text { http://dx.doi.org/10.35381/racji.v5i3.1089 }}$ 
Diario el Metro (10-noviembre de 2019) Ecuador: más de 300 mil accidentes de tránsito se registraron en los últimos 10 años. [Ecuador: more than 300,000 road accidents have been recorded in the last 10 years]. Recuperado de: https://n9.cl/qdl75

Organización Panamérica de la Salud (2017) Beber y Conducir [Drinking and Driving]. Recuperado de: https://n9.cl/xro5

Organización Mundial de la Salud (2018). El consumo nocivo de alcohol mata a más de 3 millones de personas al año, en su mayoría hombres. [Harmful alcohol consumption kills more than 3 million people a year, mostly men]. Recuperado de: https://n9.cl/hiwo4

Real Academia Española. (2019). Diccionario de la Real Academia Española. [Dictionary of the Royal Spanish Academy] Recuperado de: https://dle.rae.es/embriaguez

Sotomayor-Acosta, J. \& Álvarez-Álvarez, J. (2014) El homicidio imprudente agravado por la embriaguez o el influjo de sustancias que produzcan dependencia física o psíquica [Reckless homicide aggravated by drunkenness or influx of substances that cause physical or mental dependence]. Revista Nuevo Foro Penal. 10(83),128-166. Universidad EAFIT.

\footnotetext{
(C2020 por los autores. Este artículo es de acceso abierto y distribuido según los términos y condiciones de la licencia Creative Commons Atribución-NoComercial-Compartirlgual 4.0 Internacional (CC BY-NC-SA 4.0) (https://creativecommons.org/licenses/by-nc-sa/4.0/).
} 